\title{
Differential effects of a retention interval on latent inhibition and the habituation of an orienting response
}

\author{
GEOFFREY HALL and TODD R. SCHACHTMAN \\ University of York, York, England
}

\begin{abstract}
In Experiment 1, rats experienced presentations of a discrete visual stimulus (Stage 1) until habituation of the orienting response (OR) occurred. On a test session given after an interval of 16 days (Stage 2) the OR reappeared. For control subjects that received no Stage 1 training but presentations of the light in Stage 2, habituation persisted during the test. All subjects then received conditioning trials on which the light preceded the delivery of food. They showed latent inhibition, acquiring the conditioned response less readily than control subjects that had not previously experienced the light. Experiment 2 confirmed that the latent inhibition effect survived the retention interval for subjects that received no habituation test session. This pattern of results implies that habituation of the OR and latent inhibition are determined by different mechanisms.
\end{abstract}

Habituation has often been found to be a temporary phenomenon in that the habituated unconditioned response to a repeatedly presented stimulus will reappear when the stimulus is presented again in a test session, say $24 \mathrm{~h}$ later. Thompson and Spencer (1966) put forward such spontaneous recovery as one of the defining characteristics of habituation. Spontaneous recovery is not always complete, however (e.g., Davis, 1970; Marlin \& Miller, 1981), and with some training procedures may fail to appear when the retention interval between habituation and the test is several weeks long (e.g., Graham, 1973; Leaton, 1974). It has been suggested (e.g., Siddle, Stephenson, \& Spinks, 1983; Wagner, 1976) that a distinction should be drawn between a process of short-term habituation and one of long-term habituation and that certain training procedures (in particular the length of the interstimulus interval) might determine which of these predominates. A full account of the relevant factors remains to be presented, however: Leaton's (1974) demonstration of the long-term retention of habituation, for instance, followed training with the short interstimulus interval that might be supposed to favor a short-term effect.

When a stimulus that has been used in habituation training is used as the conditioned sitmulus in a classical conditioning paradigm, acquisition of the conditioned response proceeds slowly, an effect known as latent inhibition (Lubow, 1973). Investigations of the effects of interposing a retention interval between the preexposure and conditioning phases of a latent inhibition experiment have again revealed varied effects. Some investigators have reported perfect retention of latent inhibition (e.g., Crowell \& Anderson, 1972; James, 1971; Siegel, 1970), whereas others (e.g., Baker \& Mercier, 1982; Hall \&

\footnotetext{
This work was supported by a grant from the United Kingdom Science and Engineering Research Council. We thank K. Swann for her help. Requests for reprints may be sent to G. Hall, Department of Psychology, University of York, York, Y01 5DD, England.
}

Minor, 1984; Kraemer \& Roberts, 1984; McIntosh \& Tarpy, 1977) have found signs of a decrement. With the exception of the experiment by James (1971), these studies employed a relatively long interstimulus interval during preexposure and a retention interval of $24 \mathrm{~h}$ or more.

The experiments reported here made use of a procedure that allowed assessment both of latent inhibition and of the habituation of an unconditioned response. In this procedure (as used by Hall \& Channell, 1985), rats received a series of presentations of a discrete visual stimulus in sessions spread over several days. The initial unconditioned orienting response (OR) to the light was found to decline in frequency from session to session, a result indicative of long-term habituation. When the subjects were subsequently given training in which presentation of the light preceded the delivery of food, they developed the response of approaching the food tray during exposure to the light. This response was acquired less readily in rats given prior habituation training than in rats for which the light was novel; that is, latent inhibition occurred.

Given the results of the experiments reviewed above, it is not possible to say whether or not, in this procedure, a retention interval following the preexposure phase will be effective in restoring the habituated response and in attenuating latent inhibition. The point of importance for our purposes, however, is that certain theories about these phenomena suggest that habituation and latent inhibition should covary. According to Wagner (1976), preexposure to a stimulus can allow the formation of an association between the stimulus and the context in which it occurs. The context is then able to prime a representation of the stimulus, and a primed stimulus is less likely to receive processing; that is, it is less likely to evoke an unconditioned response and less ready to enter into an association with a reinforcer. Pearce and Hall (1980) ascribed latent inhibition to a decline in the value of a stimulus- 
specific learning-rate parameter representing stimulus associability. They have suggested (Pearce, Kaye, \& Hall, 1983; see also Hall, Kaye, \& Pearce, 1985; Kaye \& Pearce, 1984) that the frequency of occurrence of the OR might provide a direct index of the associability of the stimulus. According to both Wagner (1976) and Pearce and Hall (1980), therefore, the parameters that are effective for this training procedure in reversing habituation should also attenuate latent inhibition, and vice versa. Similarly, inappropriate parameters should be ineffective for both habituation and latent inhibition. The experiments reported here were designed to test these suggestions.

\section{EXPERIMENT 1}

There were three groups of subjects in this experiment, designated according to the treatments they received in the first two stages of training. Subjects in Group L/HC received habituation training to the light $(L)$ in Stage 1 and then remained in their home cages (HC) during Stage 2. Group HC/L received these treatments in the reverse order. Comparison of the performance of these two groups on a test session immediately following Stage 2 should reveal any effects of the retention interval for Group $\mathrm{L} / \mathrm{HC}$. The effect of these treatments on latent inhibition was examined in a final conditioning phase in which the light was made to signal food. The third group of subjects (Group HC/C) was given no preexposure to the light in Stages 1 and 2 but received equivalent experience of the training context $(C)$. The performance of this group allowed an assessment of the extent to which conditioning was retarded by light preexposure in the other two groups.

\section{Method}

Subjects. The subjects were 24 experimentally naive male hooded Lister-derived rats, maintained at $85 \%$ of their free-feeding body weights by daily weighing and controlled feeding.

Apparatus. Training was conducted in two identical Skinner boxes $(20 \times 24 \times 23 \mathrm{~cm})$ from which the response levers had been withdrawn. The boxes were housed in a small dark room and were stacked one on top of the other. With the door of the soundattenuating outer chamber left open, it was possible to observe the interior of each box through its transparent plastic door. A television camera positioned $1.2 \mathrm{~m}$ away allowed the behavior of the rat to be monitored and recorded on a videotape recorder located in a different room. Ilumination was supplied by a $30-\mathrm{W}$ striplight, operated at $60 \mathrm{~V}$, above the translucent ceiling of the box. An exhaust fan provided a constant background noise of $65 \mathrm{dBA}$ (SPL). A 3-W light bulb protruding from one of the walls of the chamber adjacent to the door was operated at $17.5 \mathrm{~V}$ to supply the light stimulus. This bulb was positioned $4.5 \mathrm{~cm}$ from the door and $10 \mathrm{~cm}$ above the floor of the chamber. Centered on the same wall at floor level was a rectangular aperture $6 \mathrm{~cm}$ high and $5 \mathrm{~cm}$ wide, protected by a hinged plastic flap, that gave access to a food tray to which $45-\mathrm{mg}$ food pellets could be delivered. A microswitch was actuated when the flap was opened.

Procedure. In the first stage of training, which lasted 16 days, subjects in Group L/HC experienced 33-min sessions each day. There were 10 presentations of a 10-sec light in each session; the mean interval between trials was 3 min (range 2-4 min). Subjects in Groups $\mathrm{HC} / \mathrm{L}$ and $\mathrm{HC} / \mathrm{C}$ remained in their home cages, un- disturbed apart from being weighed and fed each day. In the second stage, which also lasted 16 days, Group HC/L received habituation training to the light. Group HC/C was placed in the context for $33 \mathrm{~min}$ each day, but the light was not presented. Subjects in Group L/HC remained in their home cages. On the day following the completion of Stage 2 all subjects received a single test session in which the light was presented 10 times.

In preparation for the final stage of conditioning, all subjects next received two sessions of magazine training. Food pellets were delivered according to a variable-time 60-sec schedule and the rats readily learned to push back the flap in front of the food tray and retrieve the pellets. Five sessions of appetitive conditioning followed. During these sessions the light was presented just as before, but its offset accompanied the delivery of a food pellet. The number of flap openings occurring during the light and during the 10-sec period immediately preceding each trial was recorded.

The OR was scored according to the system used by Hall and Channell (1985). A critical area was marked on the screen of the video monitor corresponding to a "box" within the experimental chamber $9 \mathrm{~cm}$ long, $6.6 \mathrm{~cm}$ high, and $5.6 \mathrm{~cm}$ deep, with the light centered on one long wall. On each light presentation a record was made of the number of times the rat's snout moved into the box. An equivalent score was recorded for Group $\mathrm{HC} / \mathrm{C}$ on dummy trials during Stage 2; that is, their behavior was noted at times when a stimulus would have been presented during a habituation training session.

\section{Results}

Stages 1 and 2. Figure 1 shows the group mean performance for all sessions of habituation and the test session. The score used (following Hall \& Channell, 1985) is the number of trials per session on which at least one OR occurred. This score generates much the same pattern of results as that produced by taking the total number of ORs displayed per session. A summary of the critical results using the latter measure is presented in Table 1.

It is apparent that for the group presented with the light in Stage 1, ORs tended to occur (compare the scores on dummy trials for Group $\mathrm{HC} / \mathrm{C}$ in Stage 2), and that the frequency of occurrence of ORs declined in the second half of the stage. A comparison of Group L/HC's performance on the first and last sessions of the phase showed a significant difference on the total $O R$ score shown in Table 1 (Wilcoxon $T=2.5, N=8, p<.05$ ); the comparison using the trials score of Figure 1 fell short of significance $(T=5, N=8)$. In an attempt to reduce the effects of day-to-day variability in this measure, we computed trials-with-a-response scores for the pooled scores from the first 3 and last 3 days of Stage 1, but the difference again fell short of significance $(T=5, N=8)$.

In Stage 2, the group experiencing the light for the first time (Group HC/L) showed a pattern of orienting similar to that shown by Group L/HC in Stage 1, although for Group HC/L the decline from Day 1 to Day 16 was not statistically reliable (for trials, $T=5, N=7$; for total ORs, $T=1, N=6$ ). Again, trials-with-a-response scores were computed for Group HC/L for the pooled scores from the first 3 and the last 3 days of Stage 2. A comparison of these scores revealed a significant difference $(T=2, N=8, p<.05)$. Habituation was not complete in that the level of orienting shown by 


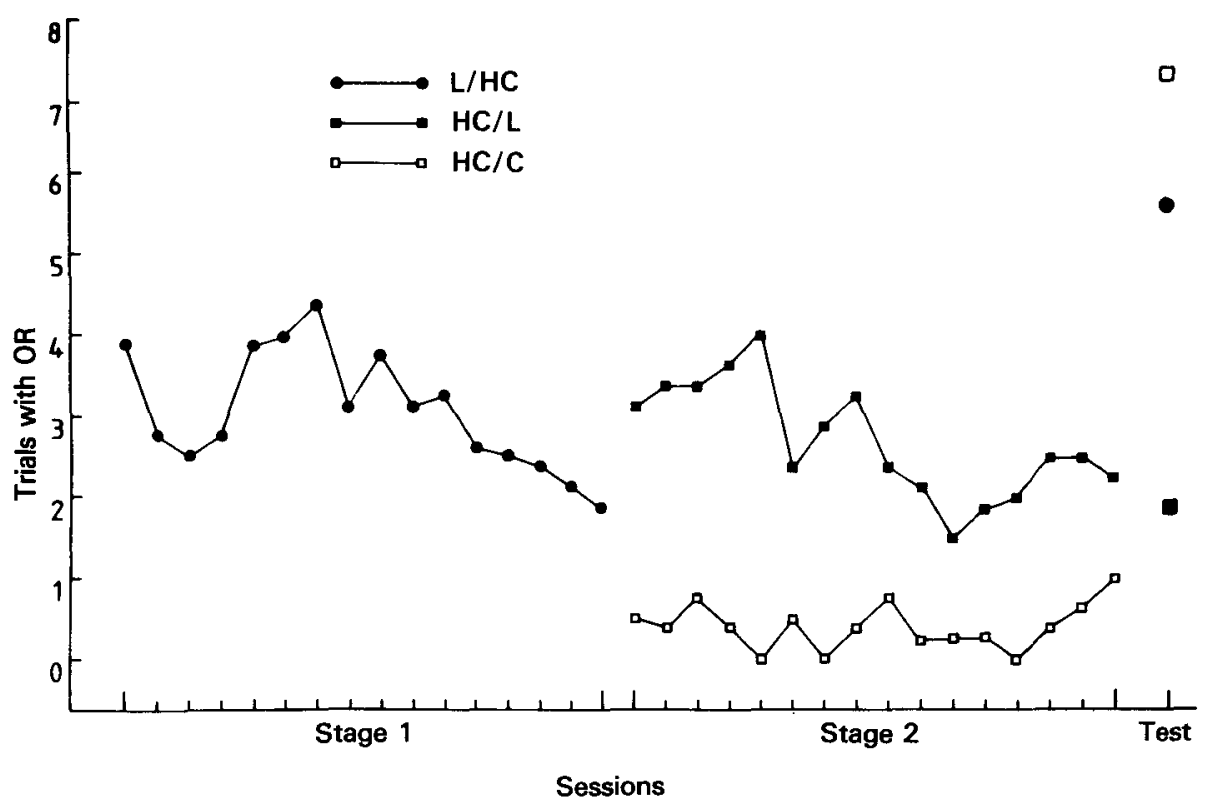

Figure 1. Experiment 1: Group mean trials per session on which an orienting response (OR) cccurred for both stages of preexposure and for the habituation test. $L$ = phase of exposure to light presentations; $\mathrm{C}=$ phase of exposure to context alone; $\mathrm{HC}=$ phase in which subjects remained in their home cages. All subjects received the light on the test session.

Group HC/L on Day 16 of Stage 2 remained numerically greater than that shown on dummy trials by the comparable control group, $\mathrm{HC} / \mathrm{C}$ (for both trials and total ORs, Mann-Whitney $U=12, p<.05)$. There was no difference, however, between the preexposed groups (HC/L and $\mathrm{L} / \mathrm{HC}$ ) in the performance they showed on the last session of habituation to the light (Day 16 of Stage 1 for Group L/HC, Day 16 of Stage 2 for Group HC/L). A comparison of the trials-with-a-response scores of these two groups yielded Mann-Whitney $U=25$, and a comparison of their total $O R$ scores yielded $U=28$.

Test session. For Group HC/L, the test session constituted a direct continuation of the previous stage of habituation training, and no change in performance was observed. A comparison of orienting on the last day of Stage 2 with that shown on the test revealed no significant differences (for trials, $T=2, N=4$; for total ORs, $T=0, N=3$ ). Both of the other groups showed a much higher level of orienting, and there was a significant difference among the three groups in analyses using both the trials score [Kruskal-Wallis $H(2)=18.56, p<.01]$ and the total-ORs score $[H(2)=20.48, p<.01]$. Further analysis showed that both of the other groups had higher trials-with-a-response scores than Group HC/L (for the

Table 1

Experiment 1: Total Orienting Responses per Session (Group Means)

\begin{tabular}{ccccccc}
\hline & \multicolumn{2}{c}{ Stage 1 } & & \multicolumn{2}{c}{ Stage 2 } & \\
\cline { 2 - 3 } Group & Day 1 & Day 16 & & Day 1 & Day 16 & Test \\
\hline L/HC & 5.63 & 2.00 & & & & 7.00 \\
HC/L & & & & 4.63 & 2.50 & 1.88 \\
HC/C & & & & 0.50 & 1.00 & 12.38 \\
\hline
\end{tabular}

Note-HC $=$ home cage; $C=$ context; $L=$ phase of exposure to the light. comparison with Group $\mathrm{HC} / \mathrm{C}, U=0, p<.01$; with Group L/HC, $U=0.5, p<.01$ ). Analysis of total ORs yielded an identical pattern of significant differences.

The performance shown by Group L/HC on the test session was not significantly different from its performance on Session 1 of Stage 1, when the light was first presented. A comparison of the group's trials scores on the two sessions yielded $T=4, N=7$, and a comparison of its total ORs yielded $T=7, N=7$. There was, however, substantially more orienting in Group L/HC on the test session than on the final day of Stage 1 habituation training (for both scores, $T=0, N=8$ ).

The test performance of Group HC/C produced higher scores than those seen in the other groups. Group HC/C's performance was significantly better than that of Group L/HC (for trials, $U=6$; for total ORs, $U=0$; ps < .05). Hall and Channell (1985) also found that the OR was particularly frequent in subjects that experienced the light for the first time after preliminary exposure to the context alone. This effect may indicate that prior exposure to the context alone allows the habituation of exploratory responses that might otherwise compete with the tendency to orient to the light.

Conditioning. All subjects readily learned to collect pellets from the food tray during magazine training. During the five conditioning sessions, they came to approach the food tray in the presence of the light, although some openings of the flap in front of the tray were also recorded in the absence of the light. The rate of response in the absence of the light was low and was similar in all groups. The mean number of responses per minute recorded in all prestimulus periods over the conditioning phase was 3.66 for Group L/HC, 3.42 for Group HC/L, and 2.86 


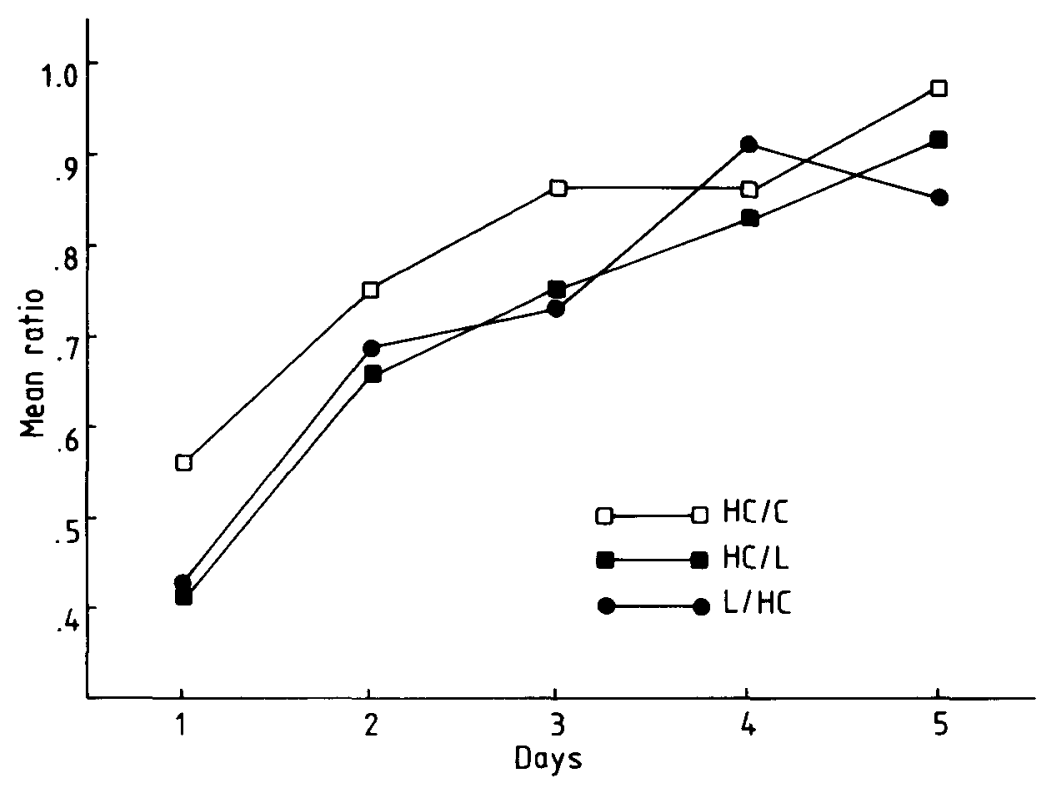

Figure 2. Experiment 2: Group mean flap entry responses during light-food pairings, expressed as a ratio of prestimulus response rate. Group designations as in Figure 1 .

for Group HC/C. There was no significant difference among these scores [Kruskal-Wallis $H(3)=0.68$ ] .

Conditioned responding was assessed by means of the ratio $a /(a+b)$, where $a$ represents the total number of flap openings recorded in the presence of light for an individual on a given session and $b$ represents the number of openings occurring during prestimulus periods on that day. Group means are presented in Figure 2. It is apparant that conditioning occurred rapidly in all groups, and, although the differences among the groups are small, there is some indication that the performance of Group $\mathrm{HC} / \mathrm{C}$ was superior to that of the other groups, which differed little from one another. Statistical analyses carried out on the mean ratio scores over all 5 days largely confirmed these impressions. A comparison of all three groups yielded Kruskal-Wallis $H(2)=6.31, .10>p>.05$. A comparison of individual pairs of means showed there to be no difference between Groups L/HC and HC/L $(U=30.5)$; however, Group HC/C differed significantly from Group L/HC $(U=11, p<.05)$ and from Group HC/L $(U=12, p<.05)$.

\section{Discussion}

These results confirm (see Hall \& Channell, 1985) that the OR initially evoked in the rat by a discrete visual stimulus will decline with repeated presentations of the stimulus, and that the low level of response persists from one session to another $24 \mathrm{~h}$ later. Thus, Group HC/L showed the same (low) level of orienting to the light on the test session as on the immediately preceding (16th) session of habituation training. Interposing a long (16-day) retention interval, however, between habituation training and the test session resulted in the frequency of the OR returning to its initial level in Group L/HC. Long-term habituation (regarded as such because of its persistence from one daily session to the next) can show spontaneous recovery, given a sufficiently long retention interval.

Latent inhibition did not show this pattern of recovery. The superiority in conditioning of Group HC/C (for which the light was relatively novel, having been experienced previously only on the test session) over Group $\mathrm{HC} / \mathrm{L}$ constitutes a demonstration of latent inhibition. But performance of Group L/HC was almost identical to that shown by Group HC/L, and thus the latent inhibition effect produced by these procedures survived intact a long retention interval spent in the home cage.

The overall pattern of the results reported here constitutes a difficulty for any theory (e.g., Pearce et al., 1983; Wagner, 1976) that proposes that latent inhibition and habituation of the OR depend upon a common mechanism. The habituated OR reappeared after the retention interval in Group L/HC, but the latent inhibition effect remained. The difficulty could perhaps be overcome if it were supposed that the restored associability implied by the high level of orienting shown by Group L/HC on test failed to carry over to the conditioning phase. It might be argued that a long retention interval allows recovery of the OR but that habituation is rapidly reacquired (and associability is lost) during the course of the test session itself. Latent inhibition might therefore be expected during the phase of conditioning that follows closely after the test session. Experiment 2 constituted a test of this argument.

\section{EXPERIMENT 2}

In this experiment the three groups of subjects received habituation training identical to that given in Stages 1 and 
2 of Experiment 1. There was no habituation test session, however, and the conditioning procedures began immediately after Stage 2 had been completed. Would Group L/HC still show latent inhibition in the absence of any opportunity (such as was provided by the test session of Experiment 1) to renew its habituation to the light?

\section{Method}

The subjects were 24 naive male hooded Lister rats, maintained at $80 \%$ of their free-feeding body weights. The apparatus and training procedures were the same as those used in Experiment 1 . Thus, there were again three groups of subjects, $\mathrm{L} / \mathrm{HC}, \mathrm{HC} / \mathrm{L}$, and $\mathrm{HC} / \mathrm{C}$, receiving 16 days of Stage 1 training and 16 days of Stage 2. After magazine training, all subjects received five sessions of conditioning with presentation of the light preceding food. In an attempt to obtain a measure of conditioning more stable than that used in Experiment 1, the score taken was the number of trials per session on which at least one opening of the magazine flap occurred. In all other respects the procedure was identical to that used in Experiment 1 .

\section{Results and Discussion}

Habituation to the light (for Groups L/HC and HC/L) proceeded much as in Experiment 1. Accordingly, Table 2 presents only the initial and terminal performance of these animals. Both groups showed a significant decline in performance from the start of habituation training to the end. A comparison of the trials score shown in Table 2 for Group L/HC yielded $T=1.5, N=7$, $p<.05$, and a comparison of the total ORs yielded $T=4, N=8, p<.05$. Equivalent tests for Group HC/L yielded $T=1, N=7, p<.05$ on both measures. The two groups did not differ in their terminal performance: for the trials score, $U=16$, and for the total ORs, $U=19$.

Table 2 also shows the orienting performance of all groups on the first day of conditioning. Because it followed a period of magazine training and was derived from trials on which food pellets were delivered, this performance cannot provide a pure measure of the effects of a retention interval on habituation. Nonetheless, the pattern of results exactly matched that recorded on the habituation test session of Experiment 1. A comparison of terminal performance in the habituation phase with that shown on Day 1 of conditioning revealed no significant

Table 2

Experiment 2: Orienting (Group Means per Sesvion)

\begin{tabular}{|c|c|c|c|}
\hline \multirow[b]{2}{*}{ Group } & \multicolumn{2}{|c|}{ Habituation } & \multirow{2}{*}{$\begin{array}{c}\text { Conditioning } \\
\text { Day } 1\end{array}$} \\
\hline & Days 1-2 & Days 15-16 & \\
\hline \multicolumn{4}{|c|}{ Trials With Orienting Response } \\
\hline $\begin{array}{l}\mathrm{L} / \mathrm{HC} \\
\mathrm{HC} / \mathrm{L} \\
\mathrm{HC} / \mathrm{C}\end{array}$ & $\begin{array}{l}4.50 \\
4.06\end{array}$ & $\begin{array}{l}2.81 \\
1.81\end{array}$ & $\begin{array}{l}5.12 \\
1.87 \\
8.62\end{array}$ \\
\hline \multicolumn{4}{|c|}{ Total Orienting Responses } \\
\hline $\begin{array}{l}\text { L/HC } \\
\text { HC/L } \\
\text { HC/C }\end{array}$ & $\begin{array}{l}6.50 \\
7.50\end{array}$ & $\begin{array}{l}3.88 \\
2.69\end{array}$ & $\begin{array}{r}6.88 \\
2.50 \\
17.38\end{array}$ \\
\hline
\end{tabular}

Note-HC = home cage; $C=$ context; $L=$ phase of exposure to the light. change in Group HC/L (for the trials score, $T=10$, $N=6$; for total ORs, $T=16, N=8$ ). Group L/HC showed an increase in orienting on both measures, an increase that fell short of statistical significance using the trials score $(T=5, N=8)$ but was reliable for total ORs $(T=2.5, N=8, p<.05)$.

The groups differed in the orienting they showed on the first conditioning day [for the trials score, KruskalWallis $H(2)=18.06, p<.01$ ]. A comparison of individual pairs of means confirmed that Group L/HC showed significantly more orienting than did Group HC/L $(U=6, p<.01)$. As in Experiment 1, Group HC/C showed more orienting than did either of the other groups (for the comparison with Group $\mathrm{HC} / \mathrm{L}, U=0, p<.01$; for the comparison with Group $\mathrm{L} / \mathrm{HC}, U=2, p<.01$ ). But although it fell short of that achieved by Group HC/C, the performance of Group L/HC on the first conditioning day was fully restored to its level at the beginning of habituation training. For Group L/HC, there was no significant difference on the trials score between the beginning of Stage 1 and the first conditioning day $(T=12.5, N=8)$. There was, however, substantially more orienting on the first conditioning day than on the last day of habituation training $(T=1, N=7)$. Equivalent analyses using the total ORs score yielded an identical pattern of significant differences.

Figure 3 shows, for the 5 days of conditioning, the mean number of trials (out of a possible 10) on which at least one opening of the magazine flap was recorded. Magazine activity increased steadily in all three groups, but the increase was most rapid in Group $\mathrm{HC} / \mathrm{C}$; that is, a latent inhibition effect was evident in the groups given preexposure to the light. There is no suggestion in the results that the retention interval produced an attenuation of latent inhibition; if anything, conditioning proceeded more slowly in Group L/HC than in Group HC/L. Statistical analyses were carried out on the scores pooled over all conditioning days. There proved to be a reliable difference among the groups [Kruskal-Wallis $H(2)=7.32, p<.05]$. Subsequent analyses showed there to be no significant difference between Groups $L / H C$ and $H C / L(U=17)$. The difference between Groups $\mathrm{HC} / \mathrm{L}$ and $\mathrm{HC} / \mathrm{C}$ also proved to be unreliable $(U=17.5)$, but there was a significant difference between Groups L/HC and HC/C $(U=8.5, p<.05)$. It is not clear why the basic latent inhibition effect (shown by the comparison of Groups HC/L and HC/C) was reduced in magnitude in this experiment, compared with that shown in Experiment 1 and that reported by Hall and Channell (1985). The important finding for our purposes, however, is that a sizable effect was evident in Group L/HC in spite of the fact that the retention interval was effective in restoring the habituated OR.

\section{GENERAL DISCUSSION}

The results reported here show that the habituated OR to a light will be restored when a long retention interval 


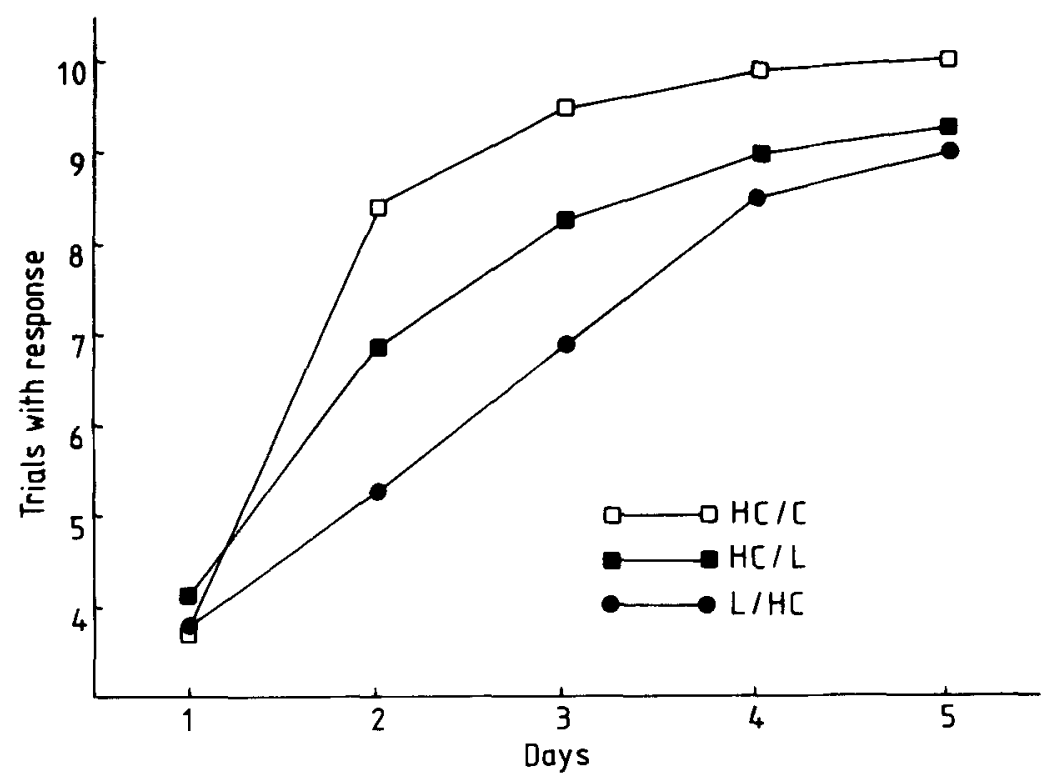

Figure 3. Experiment 3: Trials per conditioning session on which a magaxime response was recorded (group means). Group designations as in Figure 1.

spent in the home cage intervenes between habituation training and the habituation test session. Conditioning to the light proceeds only slowly, however, suggesting that the latent inhibition effect can survive the retention interval. Taken together with the results previously reported by Hall and Channell (1985), these findings constitute a double dissociation of latent inhibition and habituation of the OR.

In an investigation of the effects of contextual change on the effectiveness of a preexposed stimulus, Hall and Channell (1985) used procedures very similar to those used in the experiments reported here. Rats were given preexposure to the light in one distinctive context and habituation and conditioning were tested in a different context. Hall and Channell found (using a test context with which the animals were familiar but in which the light had not previously been presented) that the frequency of occurrence of the OR remained low. But although habituation of the $O R$ transferred from one context to another, the latent inhibition effect did not. Learning occurred slowly when light-food pairings were given in the preexposure context, but at a normal rate when conditioning was carried out in the new context.

Hall and Channell (1985) suggested that these results implied that latent inhibition and habituation are determined by independent mechanisms. But they also acknowledged another possible interpretation-that the latent inhibition procedure might simply supply a more sensitive test than does the OR of the strength of some common process that underlies both. The present results speak against this alternative interpretation. They again demonstrate a dissociation of habituation and latent inhibition, but here it is the latent inhibition effect (supposedly the more sensitive measure) that is unaffected by the procedure, whereas the habituated $O R$ is fully restored.
Some revision of current theories is required if the independence of habituation and latent inhibition is to be properly accommodated. Thus the suggestion that the frequency of orienting to a light can provide a measure of the associability of that stimulus (Pearce et al., 1983) must, at the very least, be subject to qualification. One possibility worth brief mention is that the likelihood of an OR might be determined not only by the associability of the specific stimulus, but also by the extent to which the context in which the stimulus is presented is generally arousing. Habituation of the response might depend upon a decline in both the specific and the general processes; restoration of the response might possibly be produced by an increase in the general level of arousal only (cf. the dual-process theory of, e.g., Thompson, Groves, Teyler, \& Roemer, 1973). It should be possible, according to this account, to find procedures that would restore a habituated OR but leave the associability of the stimulus relatively low. It is perhaps inappropriate to pursue this speculation in advance of further experimental evidence, but it is at least plausible to suppose that one of the effects of a long retention interval might be to restore the arousing properties of a previously experienced experimental context.

\section{REFERENCES}

BaKer, A. G., Mercier, P. (1982). Extinction of the context and latent inhibition. Leaming \& Motivation, 13, 391-416.

Crowell, C. R., ANDerson, D. C. (1972). Variations in intensity, interstimulus interval, and interval between preconditioning CS exposures and conditioning with rats. Joumal of Comparative \& Physiological Psychology, 79, 291-298.

DA vIS, M. (1970). Effects of interstimulus interval length and variability of startle-response habituation in the rat. Journal of Comparative \& Physiological Psychology, 72, 177-192.

GraHAM, F. K. (1973). Habituation and dishabituation of responses in- 
nervated by the autonomic nervous system. In H. V. S. Peeke \& M. J. Herz (Eds.), Habituation: Vol. 1. Behavioral studies (pp. 163-218). New York and London: Academic Press.

Hall, G., Channell, S. (1985). Differential effects of contextual change on latent inhibition and on the habituation of an orienting response. Joumal of Experimental Psychology: Animal Behavior Processes, 11, 470-481.

Hall, G., Kaye, H., * Pearce, J. M. (1985). Attention and conditioned inhibition. In R. R. Miller \& N. E. Spear (Eds.), Information processing in animals: Conditioned inhibition (pp. 185-207). Hillsdale, NJ: Erlbaum.

Hall, G., \& Minor, H. (1984). A search for context-stimulus associations in latent inhibition. Quarterly Joumal of Experimental Psychol ogy, 36B, 145-169.

JAmES, J. P. (1971). Latent inhibition and the preconditioningconditioning interval. Psychonomic Science, 24, 97-98.

KaYe, H., \& Pearce, J. M. (1984). The strength of the orienting response during Pavlovian conditioning. Joumal of Experimental Psy chology: Animal Behavior Processes, 10, 90-109.

Kraemer, P. J., RoberTs, W. A. (1984). The influence of flavor preexposure and test interval on conditioned taste aversions in rats. Learning \& Motivation, 15, 259-278.

LEATON, R. N. (1974). Long-term retention of the habituation of lick suppression in rats. Joumal of Comparative \& Physiological Psychology, 87, 1157-1164.

LuBow, R. E. (1973). Latent inhibition. Psychological Bulletin, 79, 398-407.

Marlin, N. A., MillzR, R. R. (1981). Associations to contextual stimuli as determinants of long-term habituation. Joumal of Experimental Psychology: Animal Behavior Processes, 7, 313-333.

MCINTOSH, S. M., \& TARPY, R. N. (1977). Retention of latent inhibi- tion in a taste-aversion paradigm. Bulletin of the Psychonomic Society, 9, 411-412.

Pearce, J. M., Hall, G. (1980). A model for Pavlovian learning Variations in the effectiveness of conditioned but not of unconditioned stimuli. Psychological Review, 87, 532-552.

Pearce, J. M., Kaye, H., * Hall, G. (1983). Predictive accuracy and stimulus associability: Development of a model of Pavlovian learning. In M. Commons, R. J. Herrnstein, \& A. R. Wagner (Eds.), Quantitative analyses of behavior (Vol. 3, pp. 241-255). Cambridge, MA: Ballinger.

Siddle, D., Stephenson, D., \& Spinks, J. A. (1983). Elicitation and habituation of the orienting response. In D. Siddle (Ed.), Orienting and habituation (pp. 109-182). Chichester: Wiley.

SizGel, S. (1970). Retention of latent inhibition. Psychonomic Science, 20, $161-162$.

Thompson, R. F., Groves, P. M., Teyler, T. J., \& Roemer, R. A. (1973). A dual-process theory of habituation: Theory and behavior. In H. V. S. Peeke \& M. J. Herz (Eds.), Habituation: Vol. 1. Behavioral studies (pp. 239-271). New York and London: Academic Press.

Thompson, R. F., \& Spencer, W. A. (1966). Habituation: A model phenomenon for the study of neuronal substrates of behavior. Psychological Review, 73, 16-43.

WAGNER, A. R. (1976). An information processing mechanism for selfgenerated or retrieval generated depression in performance. In T. J. Tighe \& R. N. Leaton (Eds.), Habituation: Perspectives from child development, animal behavior, and neurophysiology (pp. 95-128). Hillsdale, NJ: Erlbaum.

(Manuscript received August 14, 1986; revision accepted for publication October 29, 1986.) 